\title{
Multilinguales
}

\section{Assia Djebar et la réécriture de l'histoire au féminin}

\section{Nancy Ali}

\section{(2) OpenEdition}

\section{Journals}

Édition électronique

URL : http://journals.openedition.org/multilinguales/835

DOI : 10.4000/multilinguales.835

ISSN : 2335-1853

\section{Éditeur}

Université Abderrahmane Mira - Bejaia

\section{Référence électronique}

Nancy Ali, «Assia Djebar et la réécriture de l'histoire au féminin », Multilinguales [En ligne], 6 | 2015, mis en ligne le 01 décembre 2015, consulté le 17 septembre 2019. URL : http://journals.openedition.org/ multilinguales/835; DOI : 10.4000/multilinguales.835

Ce document a été généré automatiquement le 17 septembre 2019.

\section{(c) (i) (2) $\Theta$}

Multilinguales est mise à disposition selon les termes de la Licence Creative Commons Attribution -

Pas d'Utilisation Commerciale - Pas de Modification 4.0 International 


\title{
Assia Djebar et la réécriture de l'histoire au féminin
}

\author{
Nancy Ali
}

1 "Que Dieu nous protège du mot "je" "1, /a3oza bellah men kelmet ana/2, est un adage traditionnel chez les arabo-musulmans, qu'ils soient hommes ou femmes; le pronom «je» est considéré comme un signe de vanité et de préoccupation égoïste ; caractéristiques qui s'opposent aux enseignements islamiques prônant la disparition de l'individu ${ }^{3}$ dans la collectivité, pour parvenir à la prospérité et à la réussite de l'umma ou collectivité.

2 La narration à la première personne d'une femme devient encore plus suspecte, comme nous pouvons le voir dans L'Amour, la fantasia (1985), le premier volume du projet autobiographique d'Assia Djebar qu'elle appelle le quatuor; les deuxième et troisième volumes s'intitulent respectivement Ombre sultane (1987) et Vaste est la prison (1995). Parler de soi à la première personne et afficher la subjectivité féminine dans un roman sont des transgressions majeures dans la culture algérienne: " the novelist comes to autobiography fully aware that subjectivity in life and fiction are transgressions in Algerian culture » (Mortimer, $1997: 103)^{4}$.

Dès son plus jeune âge, une femme musulmane vertueuse apprend à respecter le code du silence de la hochma " c'est-à-dire, en arabe, de la "honte", en fait la pudeur » (Hornung \& Ruhe, 1998 : 90).

3 Comment peut-on écrire le soi dans une société qui met le silence à l'honneur ? Cela a conduit les écrivains algériens, comme Assia Djebar, vers l'autofiction et l'autobiographie collective, afin de brouiller les frontières entre le réel et l'imaginaire : "[...] polyphonic discourse to blur the boundaries between fiction and experience » (Mortimer, op.cit. : 103) 5

Cet espace se situe entre l'anonymat et l'expression de la subjectivité, ou selon Rocca (2003 : 3), « un espace d'expression du désir au féminin qui déstabilise la dichotomie entre la visibilité et invisibilité du corps de la femme ».

4 Comment une femme peut-elle investir cet espace, puisque hautement surveillée : 
Voilez le corps de la fille nubile. Rendez-la invisible. Transformez-la en être plus aveugle que l'aveugle, tuez en elle tout souvenir du dehors. Si elle sait écrire? Le geôlier d'un corps sans mots - et les mots sont mobiles - peut finir, lui, par dormir tranquille: il lui suffira de supprimer les fenêtres, de cadenasser l'unique portail, d'élever jusqu'au ciel un mur orbe. Si la jouvencelle écrit? Sa voix, en dépit du silence, circule. Un papier. Un chiffon froissé. Une main de servante dans le noir. Un enfant au secret. Le gardien devra veiller jour et nuit. (Djebar, 1995a : 11 12)

5 Assia Djebar commence à écrire son autobiographie, «Fille arabe allant pour la première fois à l'école, un matin d'automne, main dans la main de son père » (1995a :11), mais celle-ci devient collective, car elle y exhume l'histoire sanglante de son pays : «Ecrire ne tue pas la voix, mais la réveille, surtout pour ressusciter tant de sœurs disparues " (Djebar,1995a: 285), «Ma nuit remue de mots français, malgré les morts réveillés...(..) Et les aurores se rallument parce que j'écris » (idem. :303).

En écrivant le soi de l'intérieur d'un cadre collectif, Djebar intègre la question du féminisme et de l'identité marginalisée dans un contexte historique plus large.

Mais, c'est dans le mot étranger que Djebar capte les témoignages des femmes analphabètes du maquis. C'est grâce à cette langue de l'adversité que leurs histoires vont maintenant voir le jour ; un paradoxe qu'elle doit assumer pour que ces histoires puissent être entendues des deux côtés de la frontière linguistique : «Et l'inscription du texte étranger se renverse dans le miroir de la souffrance, me proposant son double évanescent en lettres arabes, de droite à gauche redévidées » (1995a : 69).

\section{De l'autobiographie anonyme à l'(auto)biographie collective}

Bravant l'anonymat imposé aux femmes de sa société, Djebar ose ainsi écrire son histoire personnelle, mais elle la dissimule dans la voix polyphonique des femmes qu'elle a rencontrées dans les montagnes, ainsi que de celles des femmes cloîtrées de sa famille.

En transcrivant leurs histoires dans son texte écrit, son autobiographie, Djebar compense son sentiment d'exil. Rejoignant la tradition orale, elle restaure les liens avec son passé, coupés par la colonisation française.

L'autobiographie de Djebar encadre la fenêtre à travers laquelle elle regarde les fragments de son histoire, en les compilant pour produire, à la fois, un autoportrait et la mosaïque d'une communauté.

L'Amour, la fantasia est un texte divisé en trois blocs narratifs. Le premier, « La prise de la ville ou L'amour s'écrit ", tisse des histoires de l'enfance algérienne de l'auteur, au début de la conquête française de l'Algérie en 1830.

Le second, "Les cris de la fantasia", est composé des histoires sur le colonialisme français désormais établi en Algérie, des histoires autobiographiques racontant le passage de la narratrice à l'âge d'adulte et celles de sa vie de femme mariée, de courte durée, dans un appartement à Paris.

Le dernier, «Les voix ensevelies ", le plus long, est consacré aux témoignages des femmes du Mont Chenoua qui ont participé à la révolution algérienne et qui ont été interviewées par la narratrice, pendant la guerre, à la frontière tunisienne (sujet de son film La Nouba des femmes du Mont Chenoua, 1978), et un commentaire réflexif sur la langue française et sur l'écriture de l'autobiographie d'une femme algérienne en langue étrangère. 
La juxtaposition de blocs narratifs personnels et des documents historiques dits impersonnels, ainsi que les témoignages collectifs établissent un continuum entre l'individu et le collectif dans les romans de Djebar. Le collectif dans ce roman est à la fois l'histoire écrite par les officiers français, sur la conquête de l'Algérie, et les témoignages des femmes algériennes qui avaient rejoint les maquis durant la guerre.

Le roman L'Amour, la fantasia est, en effet, souvent désigné comme une "autobiographie collective " (Geesey, 1996 : 153 167), ou une "autobiographie plurielle» (Gafaiti, 1998: 149 159), ou encore une « autobiographie au pluriel » (Déjeux op.cit. : 116), dans laquelle, à l'instar de nombreux autres textes qui ont émergé dans le Maghreb francophone, le pronom personnel «"je" de nombreux écrivains du Maghreb est donc en fait un "jenous". Le nous caché dans le "je" est opposé au "nous" de l'Occident » (Déjeux, 1994 : 66 67).

C'est ce que confirme Djebar, dans un entretien avec Lise Gauvin: "L'écriture autobiographique est forcement une écriture rétrospective où votre "je" n'est pas toujours le je, ou c'est un "je-nous" ou c'est un "je" démultiplié » (Gauvin, 1997 : 33).

Cependant, l' " autobiographie plurielle » de Djebar se déploie sous le signe du féminin (Jaouad, $19871988: 27$ ) : "What makes this "autobiographie plurielle" remarkable is that Djebar's narrative unfolds under the fecund sign of the feminine $»^{6}$. Ce qui rend l'autobiographie au pluriel de Djebar différente de celle de ses homologues francophones masculins est qu'elle écrit une autobiographie collective spécifiquement féminine : témoignages de femmes dans la guerre, histoires des femmes de sa famille.

Ainsi, au voile qui est fourni à l'auteur par l'usage de la langue française - parce que le français permet à Djebar d'installer une certaine distance avec elle-même (Gauvin, 1997 : 24) - s'ajoute celui du je-nous qui protège également l'auteur de l'exposition et de $l^{\prime}$ « indécence implicite » de l'écriture autobiographique :

Tenter l'autobiographie par les seuls mots français, c'est, sous le lent scalpel de l'autopsie à vif, montrer plus que sa peau. [...] Parler de soi-même hors de la langue des aïeules, c'est dévoiler certes, mais pas seulement pour sortir de l'enfance, pour s'en exiler définitivement. Le dévoilement, aussi contingent, devient, comme le souligne mon arabe dialectal du quotidien, vraiment "se mettre à $n u$ " [...] Me mettre à $n u$ dans cette langue me fait entretenir un danger permanent de déflagration. De l'exercice de l'autobiographie dans la langue de l'adversaire d'hier. (Djebar, 1995a : 224 300).

Prise entre la nécessité d'affirmer une voix individuelle et le désir de rattacher cette voix à la polyphonie de celles de la communauté des femmes (ses aïeules et les autres), l'autobiographie djebarienne est pour le moins paradoxale. Afin de rétablir les liens avec son passé, rompus par la colonisation et l'acculturation française, elle doit se résigner à faire parler la collectivité anonyme des femmes qui en portent témoignage. Afin de revisiter l'histoire de son pays, elle doit mêler sa voix singulière à la voix plurielle des femmes (Idem. : 234) : "Dire à mon tour. Transmettre ce qui a été dit, puis écrit. Propos d'il y a plus de un siècle, comme ceux que nous échangeons aujourd'hui, nous, femmes de la même tribu ».

En dévoilant sa voix et en refusant le silence pudique dicté par la hochma et autres traditions coercitives responsables de l'inhibition de la subjectivité des femmes, Djebar délivre donc les voix des autres femmes. Agissant en tant que lien entre les histoires des femmes, elle resitue la «trame de [leur] histoire murmurée » (Idem. : 201) et « la chaîne des femmes autrefois enfermées, génération après génération » (Djebar, 2002 : 124).

Grâce à l'écriture, elle se fait femme-conteuse à son tour et elle prend sa place dans la halqa traditionnelle (Bonn, 2004: 7) ${ }^{7}$, le cercle des conteuses-auditrices composé de femmes aux voix anonymes et aux corps entrelacés. Et comme les conteuses de la halqa 
dont Djebar fait entendre les voix, son autobiographie laisse voir la nécessité de se raconter.

Mais comment peut-elle attester de la subjectivité de son moi tout en fondant sa voix dans la collectivité des femmes? Djebar examine les problématiques de son identité singulière au pluriel prise entre affichage et camouflage (Djebar, 1995a : 91 92) : «Dévoiler et simultanément tenir secret ce qui doit le rester, tant que n'intervient pas la fulgurance de la révélation".

Alors qu'elle exprime une identité individuelle, cette identité se propage dans le récit d'ensemble, tout comme ses chapitres autobiographiques s'entremêlent au discours historique et à la polyphonie des voix féminines.

L'auteur assume ainsi le rôle d'«intermédiaire » pour faire connaître les documents historiques, les témoignages oraux, les archives françaises en se focalisant sur le rôle des femmes algériennes ou les oubliées des historiens et chroniqueurs (Fayad, 1995 : 154) : " The narrator therefore views her role as that of an intermediary, not only between her people and the French archives, but also between a male-centered history and one that focuses on the role of Algerian women in the struggle for independence $»^{8}$. Ce faisant, elle consolide la culture orale en l'ancrant irrémédiablement au monde de l'écrit; et depuis sa posture "à la jonction de plusieurs mondes - celui du père, occidentalisé, de la mère, arabo-berbère- de plusieurs temps, de plusieurs sociétés, elle se sent elle-même porteuse de mémoire, médiatrice, passeuse, scripteuse » (Perrot, 2005 : 38).

De plus, en élaborant une autobiographie collective féminine, elle ouvre son roman à l'altérité, produisant l'Autre Histoire - l'Autre des documents français de la colonisation, mais aussi l'Autre de l'homme.

Une autobiographie collective au féminin n'est donc pas nécessairement féminine en raison du sexe biologique de son auteur, mais en raison de sa capacité à donner voix au chapitre à celles qui sont réduites au silence par l'histoire dominante, à révéler l'Autre Histoire et l'histoire de l'Autre.

Le défi de la dichotomie entre l'individuel et collectif est en jeu dans toutes les œuvres du quatuor:

All three [volumes of Djebar's quartet] are polyphonic texts that combine personal and collective memory. The first and third juxtapose autobiographical fragments with Algerian history; the second replaces history with myth, recalling the legendary Sheherazade. By delving into her individual and collective past, Djebar adds her own voice to those of her maternal ancestors, both historical and legendary.

De l'aveu même d'Assia Djebar, le quatuor, dans son recours à la langue française, lie l'histoire personnelle à l'histoire collective (Djebar 1995a : 223) : « Comment une femme pourrait parler haut même en langue arabe [...] ? Comment dire "je", puisque ce serait dédaigner les formules-couvertures qui maintiennent le trajet individuel dans la résignation collective?».

L'Amour, la fantasia, Vaste est la prison et Ombre sultane sont polyphoniques en ce qu'ils comportent une multiplicité de « discours » différents : autobiographiques, historiques, mythiques, ... 


\section{Fillette arabe allant à l'école...}

7 En fréquentant une école française, Djebar avait déjà pris ses distances vis-à-vis des femmes de sa communauté, s'étant dotée d'une arme verbale qui leur avait été refusée (idem. : 11 12) :

Si elle sait écrire? Le geôlier d'un corps sans mots - et les mots écrits sont mobiles - peut finir, lui, par dormir tranquille: il lui suffira de supprimer les fenêtres, de cadenasser l'unique portail, d'élever jusqu'au ciel un mur orbe. Si la jouvencelle écrit? Sa voix, en dépit du silence, circule. Un papier. Un chiffon froissé. Une main de servante, dans le noir. Un enfant au secret. Le gardien devra veiller jour et nuit. L'écrit s'envolera par le patio, sera lancé d'une terrasse. Azur soudain trop vaste. Tout est à recommencer.

L'Amour, la fantasia s'ouvre sur l'image désormais emblématique du parcours identitaire d'Assia Djebar (idem. : 11 12) :

Fillette arabe allant pour la première fois à l'école, un matin d'automne, main dans la main du père. Celui-ci, un fez sur la tête, la silhouette haute et droite dans son costume européen, porte un cartable, il est instituteur à l'école française. Fillette arabe dans un village du Sahel algérien.(...) Les mois, les années suivantes, je me suis engloutie dans l'histoire d'amour, ou plutôt dans l'interdiction d'amour (...). Dans cette amorce d'éducation sentimentale, la correspondance secrète se fait en français: ainsi, cette langue que m'a donnée le père me devient entremetteuse (...).

Cette image de la "fillette allant à l'école » française, la "main dans la main de son père ", incarne l'histoire de l'auteur sous le signe de l'ambivalence et de la contradiction.

Parce qu'elle est allée à l'école française, elle a entrepris un parcours d'altérité, car elle s'est sentie différente à la fois des Françaises de l'école et de ses semblables, à savoir ses cousines et ses connaissances algériennes (idem. : 261) :

Ces apprentissages simultanés, mais de mode si différent, m'installent, tandis que j'approche de l'âge nubile (le choix paternel tranchera pour moi : la lumière plutôt que l'ombre) dans une dichotomie de l'espace. Je ne perçois pas que se joue l'option définitive: le dehors et le risque, au lieu de la prison de mes semblables.

Djebar reconnait la situation paradoxale dans laquelle elle se trouve : son père la libère de la prison de ses semblables en l'envoyant à l'école française, mais en même temps ses liens avec sa langue et sa culture maternelles sont rompus à cause de la conquête française et de sa politique d'acculturation et d'assimilation imposée au peuple algérien.

Djebar se sent « fugitive et ne le sachant pas, fugitive et le sachant désormais » (Djebar, $1995 \mathrm{~b}: 347)$.

Cependant, du fait de son éducation française, la fillette arabe a maîtrisé la langue qui lui a permis de circuler et de naître au monde, tout en étant écartée de ses « sœurs " voilées.

Dès son jeune âge, Djebar reconnaît le privilège qui lui a été donné grâce à ce choix paternel, sachant que la liberté qui lui a été accordée, a été refusée à ses « sœurs » (idem. : 259) :

Qu'est devenue la fille du boulanger? Voilée certainement, soustraite du jour au lendemain aux chemins de l'école: son corps la trahissait. Ses seins naissants, ses jambes qui s'affinaient, brefl'apparition de sa personnalité de femme la transforma en corps incarcéré.

Son entrée à l'école française incarne le moment où Djebar embarque pour un voyage «bilingue » et «biculturel» (Mortimer, op.cit.: 102) qui l'amène à l'écriture pour échapper à la prison du silence (Djebar, 1995a : 13 ) :

J'ai fait éclater l'espace en moi, un espace éperdu de cris sans voix, figés depuis longtemps dans une préhistoire de l'amour. Les mots une fois éclairés - ceux-là mêmes que le corps 
dévoilé découvre -, j'ai coupé les amarres. Ma fillette me tenant la main, je suis partie à l'aube.

Pour Djebar, effectivement, «le mot est torche» (Idem. : 92) pour " éclairer le corps pour aider à lever l'interdit... Dévoiler et simultanément tenir secret ce qui doit le rester, tant que n'intervient pas la fulgurance de la révélation » (Idem. : 91 92). Mais

(se) dévoiler dans la langue de l'autre c'est aussi connaître l'exil (idem. : 224) :

Parler de soi-même hors de la langue des aïeules, c'est se dévoiler certes, mais pas seulement pour sortir de l'enfance, pour s'en exiler définitivement. Le dévoilement, aussi contingent, devient, comme le souligne mon mot arabe dialectal du quotidien, vraiment "se mettre à nu".

Ainsi, pour Djebar, la liberté, qui lui a été donnée par la fréquentation de l'école française, est hantée par «weight of exile» (Mortimer, op.cit. : 102) ${ }^{10}$. Dans L'Amour, la fantasia, l'écrivaine décrit ce sentiment d'exil pour le charger de son " poids " (Djebar, 1995a : 303) : "On me dit exilée. La différence est plus lourde : je suis expulsée de là-bas pour entendre et ramener à mes parents les traces de la liberté... je crois faire le lien, je ne fais que patouiller, dans un marécage qui s'éclaire à peine ».

Cependant, parce qu'elle s'écrit, Djebar renverse la malédiction de l'anonymat qui pesait sur elle-même et sur toutes les autres femmes algériennes (Djebar, 1995a : 255) :

Comme si soudain la langue française avait des yeux, et qu'elle me les ait donnés pour voir dans la liberté, comme si la langue française aveuglait les mâles voyeurs de mon clan et qu'à ce prix, je puisse circuler, dégringoler toutes les rues, annexer le dehors pour mes compagnes cloîtrées, pour mes aïeules mortes bien avant le tombeau.

Si pour "savoir écrire " cette langue, "la fillette arabe" avait la "main dans la main du père ", lorsque, plus tard, elle se met à " écrire ", mêlant sa propre autobiographie aux histoires occultées de ses compagnes et aïeules, elle invoque Eugène Fromentin qui l'«a accompagnée en seconde silhouette paternelle » (Djebar, 1995a : 313) :

Eugène Fromentin me tend une main inattendue, celle d'une inconnue qu'il n'a jamais pu dessiner. En juin 1853, lorsqu'il quitte le Sahel pour descendre aux portes du désert, il visite Laghouat occupée après un terrible siège. Il évoque alors un détail sinistre: au sortir de l'oasis que le massacre, six mois après, empuantit, Fromentin ramasse, dans la poussière, une main coupée d'Algérienne anonyme. Il la jette ensuite sur son chemin. Plus tard, je me saisis de cette main vivante, main de la mutilation et du souvenir et je tente de lui faire porter le "qalam".

La continuité établie entre l'image du père tenant sa fillette par la main pour aller à l'école française, à l'incipit du roman, et celle de la main (coupée) de la femme que le peintre Fromentin tend à l'écrivaine à l'excipit, " est symboliquement restaurée et les échos se multiplient entre la mémoire, le savoir et la création artistique »(Chikhi, 1997 : 172).

La fillette arabe entre dans un dialogue imaginaire avec la main mutilée de l'Algérienne anonyme, décrite par Fromentin dans Un été au Sahara, en 1852.

La «main » est donc le fil qui tisse les uns aux autres les fragments narratifs de ce roman, autorisant la mise au jour de sa symbolique : la main qui a libéré la «fillette arabe allant » à l'école française, la main qui a enseigné le français à la fillette arabe, la main qui a été détachée du cadavre mutilé et la main qui écrit sont explicitement associées pour constituer la grande métaphore de l'écriture de la femme en Algérie.

D'une part, la main amputée que Fromentin décrit et peint, la main anonyme dont Djebar se saisit, est un symbole de l'Algérie amputée par une histoire écrite des mains des envahisseurs français, et d'autre part, celles des historiens masculins, des écrivains, des artistes qui ont occulté les femmes (Marx-Scouras, 1993 : 176).

En effet, cet effacement des femmes du discours de l'Histoire de l'Algérie revient en leitmotiv dans l'œuvre de Djebar en général et de L'Amour, la fantasia en particulier. 
La « main enfantine » (Djebar, 1995a : 260) de la fillette arabe prend le qalam pour écrire l'histoire de son pays avec le point de vue féminin. Dans le même temps, cette " main » donne le qalam à l'Algérienne anonyme pour qu'elle puisse s'écrire dans l'histoire de son pays (Idem. : 313) : «Plus tard, je me saisis de cette main vivante, main de la mutilation et du souvenir et je tente de lui faire porter le "qalam" ».

Cette main coupée, que Djebar donne à ses sœurs proverbialement, en transcrivant leurs témoignages, devient le symbole de la libération des femmes et de la nécessité pour elles de raconter leurs histoires.

En réécrivant l'histoire de son pays, Djebar raccorde leurs mains coupées à leurs origines algériennes, ressuscitant le passé dans son texte.

N'étant plus anonyme, l'Algérienne vient à la vie, car elle écrit. Tout comme Fromentin a tenu la main à Djebar, Djebar donne la parole aux femmes dont elle déterre les « voix ensevelies » dans la troisième partie du roman.

Par cette anamnèse, Djebar compense "l'amnésie » des documents français et de ceux écrits par les hommes arabes qu'elle dénonce à maints endroits de son texte.

Ce geste salvateur d'Assia Djebar, qui consiste à doter la femme de sa société d'« armemot $»^{11}$, ou encore de " mot-torche $»^{12}$, se décline en plusieurs scènes symboliques dans son œuvre: "Je tiens la main de ma fillette, je la tire au soleil, je l'aiderai, elle, à ne pas s'engloutir!» (Djebar, 2006 : 100).

Ainsi, dans Ombre sultane par exemple, Isma donne à Hajila la clé de l'appartementrefuge pour lui offrir une chance d'échapper à l'esclavage domestique (idem. : 204 205) :

Je me penchai pour t'embrasser. La vieille servante qui entrait pensa, c'est normal, que je te donnais le protocolaire baiser avec bénédiction, c'est le rite de la sortie du hammam... Mais moi, je chuchotai comme si, définitivement, tous les déchets du passé avaient disparu. S'étaient noyés. - Voici, prends cette clef que je tiens là! Prends! [..] Sors seulement pour sortir! [.. ['est ta clef!

A ces moyens symboles de libération pour la femme, que sont la plume et la clef, il faut y ajouter l'objectif de la caméra. Dans Vaste est la prison (op. cit. : 174), la narratrice évoque le tournage de La Nouba des femmes du Mont Chenoua (op.cit.) pour insister sur la portée de la manipulation de la caméra par les femmes: elles ne sont plus observées mais contrôlent l'objectif, regardant librement le monde (Djebar 1995b : 174) :

Silhouette unique de femme, rassemblant dans les pans de son linge-linceul les quelque cinq cents millions de ségréguées du monde islamique, c'est elle soudain qui regarde, mais derrière la caméra, elle qui, par un trou libre dans une face masquée, dévore le monde.

Pour Djebar, ce n'est pas l'image de la femme «créée par le médium » qui doit être subvertie, mais «le médium créant l'image» (Leservot, 2007: 173) lui-même. Ce médium, contrôlé par les hommes, qui opprime la femme, quel que soit le regard, orientalisant ou arabo-islamique, vise à dominer les femmes. Contre le regard masculin, la femme doit se réapproprier son regard féminin.

La plume, la clé, et l'objectif féminin de la caméra consacrent le projet féministe de Djebar, fondé sur la réappropriation de la langue, de l'espace, du regard, et de l'histoire (Mortimer, op.cit. : 115).

Dans l'optique d'Assia Djebar, donner la parole ou la caméra aux femmes, c'est lancer un défi non seulement au discours colonial écrit par les Français, mais aussi à celui de l'histoire patriarcale, écrit par les Algériens de sexe masculin. 


\section{Palimpseste au féminin ou féminisation de l'histoire}

Dans le roman L'amour, la fantasia, Djebar souligne à quel point les documents historiques sont essentiellement français et masculins. Parlant au nom des femmes analphabètes de son enfance, Djebar explique leur absence de la scène de l'écriture de l'Histoire par l'ostracisme dont elles ont été victimes (Djebar, 1995a : 212) : « Hélas! nous sommes des analphabètes. Nous ne laissons pas de récits de ce que nous avons enduré et vécu!... Tu en vois d'autres qui ont passé leur temps accroupis dans des trous, et qui, ensuite, ont raconté ce qu'ils ont raconté!».

Djebar intervient pour restituer aux discours «légitimes» de l'Histoire leur part féminine, comme dans les citations suivantes:

"Je reconstitue à mon tour cette nuit - "une nuit de cannibales", dira un certain P. Christian, un médecin qui a vagabondé du camp français au camp algérien pendant la trêve de 1837 à $1839 »$ (Idem. :103).

«Dire à mon tour. Transmettre ce qui a été dit, puis écrit 》 (Idem. : 234)

"Je le lis à mon tour, lectrice de hasard, comme si je me retrouvais enveloppée du voile ancestral; seul mon cil libre allant et venant sur les pages, où ne s'inscrit pas seulement ce que le témoin voit, ni ce qu'il écoute» (Idem. :293).

Pour inverser les effets de l'amnésie qui frappe le discours historique et le discours patriarcal qui falsifient l'histoire de la colonisation et de la guerre de libération, Djebar entreprend un travail d'anamnèse pour « défouir » les histoires de ses « compagnes » et de ses « aïeules » pour les inscrire dans la grande Histoire.

La troisième partie du roman L'Amour, la fantasia s'intitule significativement Voix ensevelies.

A travers la formule "à mon tour ", reprise plusieurs fois dans le roman L'Amour, la fantasia, comme dans les extraits ci-dessus, la narratrice suit un processus de relecture et de réécriture- de surlecture et surécriture - des documents de l'histoire officielle (Donadey, 1998, 104).

L'épisode colonial des « enfumades » de plus d'un millier d'Algériens dans les grottes de Nacmaria, le 20 juin 1845, consigné par le colonel Pélissier dans un rapport au général Bugeaud, en est un exemple (Djebar, 1995a : 113) : «Près d'un siècle et demi après Pélissier et Saint-Arnaud, je m'exerce à une spéléologie bien particulière, puisque je m'agrippe aux arêtes des mots français - rapports, narration, témoignages du passé».

Ainsi, Djebar revisite les archives-palimpsestes de l'Histoire de l'Algérie pour en faire des «palimpsestes de palimpsestes » (Idem. : 115):

Pélissier, l'intercesseur de cette mort longue, pour mille cinq cents cadavres sous El Kantara, avec leurs troupeaux bêlant indéfiniment au trépas, me tend son rapport et je reçois ce palimpseste pour y inscrire à mon tour la passion calcinée des ancêtres.

Convoquant les documents français, comme celui de Pélissier, l'écriture de Djebar œuvre contre eux. Mais, elle comprend aussi que c'est paradoxalement la langue française, une langue qui «s'appuie sur la mort des [s]iens, [qui] plonge ses racines dans les cadavres des vaincus de la conquête" (Djebar, 1999: 14), qui sera en mesure de lui permettre de renouer avec le passé historique algérien (Djebar, 1995a, 16 17) :

"Qui le dira, qui l'écrira? (...) A mon tour, j'écris dans sa langue, mais plus de cinquante ans après. (...) Que se disent les femmes de la ville (...) ?».

Cet appel révèle les deux niveaux de travail du palimpseste de Djebar.

Le premier est celui des interrogations des femmes quant aux témoignages et à l'écriture de l'Histoire; en d'autres termes, "comment la mémoire algérienne peut être prise en compte dans un contexte d'effacement historique " (Donadey, op. cit. 105). Djebar 
répond en quelque sorte en fournissant, fictivement, la version de l'histoire du point de vue des Algériens.

Le second se situe au niveau des auteurs des interrogations : «... que se disent les femmes de la ville » et qui annonce que Djebar va s'intéresser davantage aux témoignages des femmes, absentes des documents historiques.

Grâce à son écriture palimpseste ${ }^{13}$, conçue comme une entreprise d'exploration et d'excavation de l'histoire à travers son œuvre, Djebar recrée un espace-temps fictif qui restaure la voix de la femme, toujours occultée (Kelly, $2000: 25$ ).

En insérant les images et les histoires de femmes dans l'histoire coloniale de l'Algérie, Djebar fait ressortir le moi et le corps féminins, enterrés par la violence coloniale et la société patriarcale. Djebar entreprend ainsi une féminisation de l'Histoire.

Son histoire féminisée donne la parole à plusieurs voix, ouvre ses frontières aux marginalisés, incorpore le son hétéroglossique du collectif féminin.

L'entreprise spéléologique de la contre-histoire féminine de Djebar repose principalement sur la transmission de deux types d'histoires. Le premier est celui des histoires de la lignée maternelle. Djebar rassemble les histoires «oubliées» de son enfance, en rapport avec ses parents et les membres féminins de sa famille.

Le second est celui de la réécriture de l'histoire nationale, qui s'inspire principalement des témoignages des femmes ayant participé à la guerre de libération.

Pour accréditer leurs voix, l'auteur réaffirme la validité et la valeur de la tradition orale négligée dans les documents officiels reposant davantage sur l'écrit : «la voix déleste la mémoire » (Djebar, 1995a : 202), « les chuchotements des femmes » (Idem. : 250) comblent les lacunes de la lignée généalogique masculine, cette «fosse commune de l'oubli (Idem.: 59).

Ce faisant, Djebar met en évidence le rôle capital de ces conteuses dans la pérennité de l'histoire de son pays par transmission orale (Idem. : 201) :

La voix raconte? Même pas. Elle débusque la révolte ancienne. La courbe des collines brûlées tant de fois se déploie, le récit déroule la chevauchée à travers les étendues rousses de ces monts appauvris, où je circule aujourd'hui. (...) La conteuse demeure assise au centre d'une chambre obscure, peuplée d'enfants accroupis, aux yeux luisants: nous nous trouvons au coeur d'une orangeraie du Tell... La voix lance ses filets loin de tant d'années escaladées, la paix soudain comme un plomb.

La mémoire de ces femmes analphabètes qui peuplent l'enfance de Djebar ne se limite pas à celle d'une femme en particulier, mais concerne tout le collectif des compagnes et des aïeules dont les voix se confondent (Djebar, 2002: 257) : «Comme si la mère, reculant en deçà de la procréation, nous masquait son corps, afin de revenir comme voix d'aïeule indéfinie, chœur intemporel où se redit l'histoire. Mais une histoire dont s'expulse l'image archétypale du corps féminin ».

La voix individuelle de la femme se fond dans la polyphonie des "voix ensevelies" (Djebar, 1995a : troisième partie) et des «corps entrelacés» (Djebar, 1995a: titres de plusieurs chapitres de la troisième partie).

Ces «voix ensevelies » et ces «corps entrelacés " parlent ensemble afin de raconter l'Autre de l'Histoire qui enracine le présent algérien dans son passé sans pour autant garantir l'émancipation de la femme (Djebar, 1995a : 252) : «Chaîne de souvenirs: n'estelle pas justement "chaîne" qui entrave autant qu'elle enracine?"

Donnant voix à ces mémoires de femmes, Djebar va au-delà de son récit autobiographique. Pour Djebar, ces femmes-conteuses ont le pouvoir de délivrer ce qu'elle appelle une histoire souterraine. 
Contre l'Histoire de l'Algérie fondée sur l'effacement du point de vue féminin, elle propose ainsi une histoire en palimpseste qui restitue l'histoire occultée des femmes qui ne savent pas écrire, une contre-histoire féminine qui subvertit l'Histoire monolithique et totalisante.

\section{BIBLIOGRAPHIE}

BONN, Charles (dir.), Échanges et mutations des modèles littéraires entre Europe et Algérie, Tome 2, Actes du colloque "Paroles déplacées" (LERTEC, Université Lumière/Lyon2), Paris, L'Harmattan, 2004.

CHIKHI, Beïda, Littérature Algérienne : Désir d'histoire et esthétique, Paris, L'Harmattan, 1997.

DÉJEUX, Jean, La littérature féminine de langue française au Maghreb, Karthala éditions, 1994.

DJEBAR, Assia, Ces Voix qui m'assiègent : en marge de ma francophonie, Paris, Editions Albin Michel, 1999.

DJEBAR, Assia, Femmes d'Alger dans leur appartement, Paris, Editions Albin Michel, 2002.

DJEBAR, Assia, L'Amour, la fantasia, Paris, Editions Albin Michel, 1995a.

DJEBAR, Assia, Vaste est la prison, Paris, Editions Albin Michel, 1995b.

DJEBAR, ASSIA, Ombre Sultane, Paris, Editions Albin Michel, 2006. DONADEY, Anne, « Elle a rallumé le vif du passé : L'écriture-palimpseste d'Assia Djebar », dans Alfred Hornung et Ernstpeter Ruhe éds., Postcolonialisme \& Autobiographie, Amsterdam, Editions Rodopi B.V., 1998, pp. 101115.

FAYAD, Mona. "Reinscribing identity : nation and community in Arab women's writing." College Literature, vol. 22, No. 1, Third World Women's Inscriptions (Feb., 1995), pp. 147160.

GAFAITI, Hafid, "L'autobiographie plurielle : Assia Djebar, les femmes et l'histoire", dans Postcolonialisme \& Autobiographie, Amsterdam, Editions Rodopi B.V., 1998, pp. 149159.

GAUVIN, Lise, L'écrivain francophone à la croisée des langues : Entretiens, Paris, Editions Karthala, 1997.

GEESEY, Patricia, "Collective Autobiography : Algerian Women and History in Assia Djebar's L'amour, la fantasia", in Dalhousie French Studies, 1996.

HORNUNG A., RUHE, E., (eds.), Postcolonialisme et Autobiographie, Amsterdam, Editions Rodopi B.V., 1998.

JAOUAD, Hedi A. "L'Amour, la fantasia : Autobiography as Fiction", CELFAN Review 7 (1987 1988), Philadelphia, Centres d'Etudes (CELFAN),

KELLY, Debra, "Explorations/excavations of History : Knowledge, liberation and the quest of the writer in Assia Djebar's L'Amour, la fantasia and Vaste est la prison, La Chouette, 2000, p. 1925.

LESERVOT, Typhaine, Le corps mondialisé : Marie Redonnet, Maryse Condé, Assia Djebar, Paris, L'Harmattan, 2007. 
MORTIMER, Mildred, “Assia Djebar's "Algerian Quartet :" A Study in Fragmented Autobiography", in Research in African Literatures, vol. 28, No. 2, Autobiography and African Literature (Summer, 1997), pp. 102-117.

PERROT, Michelle, « Histoire et mémoire des femmes dans l'œuvre d'Assia Djebar », dans Mireille Calle-Gruber (dir.), Assia Djebar, Nomade entre les murs...Pour une poétique transfrontalière,

Maisonneuve \& Larose, 2005, pp. 3342.

RoCCA, Anna, Assia Djebar. Le Corps Invisible : Voir Sans Etre Vue, 2003. Consultable sur le site : http://etd.lsu.edu/docs/available/etd-0522103115259/unrestricted/Rocca_dis.pdf - https:// digitalcommons.lsu.edu/cgi/viewcontent.cgi?article $=4152 \&$ context=gradschool_dissertations SCOURAS, Danielle M., "Muffled Screams/Stifled Voices", in Yale French Studies, 1993, pp. 82172.

\section{NOTES}

1. Jean Déjeux a ainsi intitulé le chapitre 4 de son livre, La Littérature féminine de langue française au Maghreb (Kathala Ed., 1994), qui s'ouvre sur l'explication de la formule : "Dire "je" dans un contexte arabo-musulman n'allait pas de soi il y a quelques décennies.(...) L'analyse intime du soi y est rare, sauf dans les récits où les mystiques parlent de leur expérience religieuse » (p.61).

2. Transcription phonétique de la locution lexicalisée en arabe.

3. Voir à ce sujet Abdallah Bounfour, « Autobiographie, genres et croisement des cultures : le cas de la littérature francophone du Maghreb ", in Itinéraires et contacts de cultures, $\mathrm{N}^{\circ} 10$, colloque Jacqueline Arnaud, T.1, Harmattan/Paris 13, $1^{\mathrm{er}}$ semestre 1990, pp. 85 90. Disponible sur le site : <http://www.limag.com/Textes/Iti10/Abdallah\%20BOUNFOUR.htm>.

4. «L'auteure se lance sur une écriture autobiographique bien consciente que la subjectivité dans la vie ainsi que dans la fiction sont des transgressions dans la culture algérienne. " [Notre traduction].

5. «[..] un discours polyphonique afin de brouiller les frontières entre fiction et expérience » [Notre traduction].

6. «Ce qui fait que cette autobiographie plurielle est remarquable c'est que le récit de Djebar se déroule sous le signe fécond du féminin ». [Notre traduction].

7. «L'oralité est traditionnellement le mode d'expression le plus localisé, puisqu'elle ne prend véritablement la plénitude de sa signification que dans l'interaction complexe entre les participants à la "halqa" : ce cercle d'auditeurs qui se constitue autour du conteur ou de la conteuse, et dont tous les membres se connaissent entre eux » (Bonn, 2004 : 7).

8. «L'auteure se considère comme un intermédiaire, pas seulement entre son peuple et les archives françaises, mais aussi entre une Histoire axée sur l'homme et une autre qui focalise sur le rôle joué par la femme algérienne dans la lutte de l'indépendance ». [Notre traduction].

9. « Tous trois [volumes du quatuor] sont des récits polyphoniques qui joignent la mémoire individuelle et collective. Le premier et le troisième juxtaposent des fragments autobiographiques avec l'histoire algérienne, le deuxième remplace l'histoire avec le mythe, rappelant la légende de Shéhérazade. En plongeant dans son passé personnel et collectif, Djebar ajoute sa propre voix à celles de ses ancêtres maternels, à la fois historiques et légendaires ». [Notre traduction].

10. « Le poids de l'exil ». [Notre traduction].

11. «Le mot deviendra l'arme par excellence » (Djebar, 1995a : 67).

12. «Le mot est torche» (Djebar, 1995a : 92).

13. Nous reconduisons ici le sens qu'Anne Donadey a conféré à cette expression à l'endroit de l'écriture d'Assia Djebar, dans son étude « Elle a rallumé le vif du passé : l'écriture palimpseste 
d'Assia Djebar » : " Tout au long de L'Amour, la fantasia, Assia Djebar établit une relation de palimpseste entre la récriture des archives de la colonisation française et l'utilisation de la tradition orale féminine, afin de restituer les événements datant du siècle dernier ", dans Hornung A. et Ruhe E. eds., Postcolonialisme et autobiographie, op. cit., pp. 101115.

\section{RÉSUMÉS}

Dans L'Amour, la fantasia, Assia Djebar tend la main aux sans-voix, les femmes analphabètes de la guerre en Algérie, transmettant leurs récits oraux dans son écrit en français. Nous montrerons que, d'une autobiographie anonyme à une autobiographie plurielle, Djebar négocie un lieu spécifique pour l'autobiographie féminine écrite, dans une société traditionnelle inhibitrice du «je» de la femme. Ainsi, nous nous sommes intéressée à sa posture de «conteuse » : revisitant les archives françaises de la conquête de l'Algérie, elle donne une voix aux témoignages des femmes autrement silencieuses. Nous étudierons la façon dont Djebar écrit pour naître au monde, mais aussi pour aider les autres femmes à naître au monde en inscrivant leurs récits dans le discours de l'Histoire. Ce faisant, elle réécrit l'Histoire en un palimpseste au féminin.

Assia Djebar and the Rewriting of the History in the Feminine

In L'Amour, la fantasia, Assia Djebar lends a hand to the voiceless, the illiterate women of the Algerian war, transmitting their oral testimonies in her French text. We argue that Djebar negotiates a specific place between an anonymous autobiography and a collective one for the female autobiography written in a society that frowns upon the first-person pronoun of a woman. We address the author's role as a storyteller: revisiting the French archives of the conquest of Algeria, Djebar gives a voice to the stories of the otherwise silenced women. We explore how writing for Djebar is a means of coming to life, not just for herself, but also for the women of Algeria whose stories she inscribes in the narrative of History. So doing, she rewrites History into a feminized palimpsest.

\section{INDEX}

Mots-clés : autobiographie collective, autofiction, femmes-conteuses, littérature postcoloniale, l'oralité, féminisation

Keywords : collective autobiography, autofiction, storytellers, postcolonial literature, orality, feminisation

\section{AUTEUR}

\section{NANCY ALI}

Centre de Recherche en Littérature Comparée La Sorbonne-Paris IV Paris - France 